\title{
Statistical Challenges in the Photometric Calibration for 21st Century Cosmology: The J-PAS case.
}

\author{
Jesús Varela ${ }^{1}$, David Cristóbal-Hornillos ${ }^{1}$, Javier Cenarro ${ }^{1}$, Alessadro \\ Ederoclite $^{1}$, David Muniesa ${ }^{1}$, Héctor Vázquez Ramió ${ }^{1}$, Nicolas Gruel ${ }^{2}$ \\ \& Mariano Moles ${ }^{1}$ \\ ${ }^{1}$ Centro de Estudios de Física del Cosmos de Aragón, Plaza San Juan 1, Planta 2 \\ E-44001, Teruel, Spain N \\ email: jvarela@cefca.es \\ ${ }^{2}$ University of Sheffield, Department of Physics and Astronomy, Hicks Building, Hounsfield \\ Road, Sheffield, S3 7RH, United Kingdom
}

\begin{abstract}
The success of many cosmological surveys in the near future is highly grounded on the quality of their photometry. The Javalambre-PAU Astrophysical Survey (J-PAS) will image more than $8500 \mathrm{deg}^{2}$ of the Northern Sky Hemisphere in 54 narrow +2 medium/broad optical bands plus Sloan $\mathrm{u}, \mathrm{g}$ and $\mathrm{r}$ bands. The main goal of J-PAS is to provide the best constrains on the cosmological parameters before the arrival of projects like Euclid or LSST. To achieve this goal the uncertainty in photo-z cannot be larger than $0.3 \%$ for several millions of galaxies and this is highly dependent on the photometric accuracy.

The photometric calibration of J-PAS will imply the intensive use of huge amounts of data and the use of statistical tools is unavoidable. Here, we present some of the key steps in the photometric calibration of J-PAS that will demand a suitable statistical approach.
\end{abstract}

Keywords. Cosmology: observations, techniques: photometric, methods: data analysis

\section{Introduction}

The Javalambre-PAU (Physics of the Accelerating Universe) Astrophysical Survey (J-PAS, Benítez et al. 2014) is an astrophysical survey that will image more than $8500 \mathrm{deg}^{2}$ of the Northern Sky Hemisphere in a particular filter system made of 54 narrow $(F W H M \sim 130 \AA$ ) contiguous optical bands (from $\sim 3700 \AA$ to $\sim 9150 \AA$ ) ) and two additional wider bands in the blue and red extremes. The filter system is complemented with a special $u$ filter and the standard Sloan $g$ and $r$.

The J-PAS filter system has been designed to be optimal in the determination of photometric redshifts with a goal of $\delta_{z} /(1+z) \sim 0.3 \%$ for more than 100 million galaxies, an accuracy required to improve the constrains on the cosmological parameters.

\section{The Photometric Calibration Statistical Challenges}

The photometric calibration of J-PAS will be done as follows (Varela et al. 2013):

- A previous survey (Javalambre-Photometric Local Universe Survey, J-PLUS) of the same area of the sky will be carried out with a large field of view $83 \mathrm{~cm}$ telescope using a special set of 12 filters designed to better estimate the spectral type of the stars.

- J-PLUS images will be calibrated using the extinction measurements obtained by an extinction monitor, and the observation of Primary Spectrophotometric Standard Stars. 
- We will identify the stars with $\mathrm{S} / \mathrm{N}>50$ in the 12 bands (Secondary Standard Stars, SSSs).

- The photometry of the SSSs will be fitted using libraries of stellar spectra.

- From the best fitted stellar spectra, we will compute the synthetic J-PAS photometry of the SSSs.

- On the J-PAS images, we will identify the SSSs, and comparing the observed instrumental magnitudes with the synthetic magnitudes we will provide a preliminary photometric calibration of the J-PAS images.

- Taking advantage of the high degree of overlapping between different exposures of the same filters and using the spectra of many spectrophotometric stars in the area of J-PAS, we will perform a spectro-übercalibration, that will help to improve not only the relative calibration for a single band but also among the different bands of the survey.

The large amount of the data that we will manage and the accuracy level that we require for the final photometry implies the constant use of statistical tools.

Following we present some of the "statistical challenges" that we need to face in the quest to achieve the best photometry and the best photometric redshifts required by the 21st Century Cosmology.

\subsection{Challenge 1. Extinction coefficients}

The atmospheric extinction for J-PAS will be measured with a dedicated extinction monitor. This will observed 10 optical bands that will allow to derive an atmospheric extinction curve (see Challenge 2). The measurement of the extinction in each single band will be done following the traditional procedure of observing the same star or group of stars at different airmasses. Given a star $s$ with measurements $\left\{m_{s, i}\right\}$ at airmasses $x_{i}$, the magnitude outside the atmosphere $m_{s}^{o}$ is given by the expression $m_{s}^{o}=m_{s, i}+k_{n} x_{i}$, where $k_{n}$ is the extinction coefficient in the given band for the night $n$.

In the case of a dedicated telescope, it is possible to relate the observations in different nights if there are stars that are observed in common between different nights. In that case, and assuming the stability of the optical system (ie. that the zero point doesn't change), $m_{s}^{o}$ can be considered constant and it is possible to perform a fit using data from more than one night obtaining more robust values of $k_{n}$ and $m_{s}^{o}$.

\subsection{Challenge 2. Atmospheric Extinction Curve}

Since the extinction monitor is observing in bands different from those used in J-PLUS and J-PAS, we need to construct an atmospheric extinction curve from which estimate the atmospheric extinction in any optical band.

This will be done assuming a model of the atmosphere of 3 components (Hayes \& Latham 1975): Rayleigh scattering, Ozone absorption and aerosol scattering. The general form of such model would be $\kappa(\lambda)=A_{R} \lambda^{-\alpha_{R}}+A_{O} \kappa_{O}(\lambda)+A_{a e} \lambda^{-\alpha_{a e}}$, where $\kappa(\lambda)$ is the atmospheric extinction at wavelength $\lambda, \kappa_{O}(\lambda)$ is a normalized atmospheric extinction by ozone, $\alpha_{R}$ and $\alpha_{e a}$ provide the wavelength dependence of the Rayleigh and aerosol scattering, respectively, and, finally, $A_{R}, A_{O}$ and $A_{e a}$ give the amplitude of the extinction for each component. While the normalized wavelength dependence of the absorption of each component (ie., $\lambda^{-\alpha_{R}}, \kappa_{O}(\lambda)$ and $\lambda^{-\alpha_{a e}}$ ) can be considered constant given a location, their amplitudes depend on the atmospheric conditions and are the values that we are interested on estimating.

The estimation of the amplitudes is done performing a fitting of the observed extinction coefficients obtained in Challenge 1 , with the values of the model given by $\kappa(\lambda)$, convolved with the transmission of the system in each band. 


\subsection{Challenge 3. PSF Homogenization}

The PSF homogenization needed to obtain consistent photometry between bands and to combine single exposures obtained in different seeing conditions will be performed using a combination of the PSFEx software (Bertin 2011) and software developed by our group.

This procedure will be performed in $\sim 10^{7}$ single exposures to produce $\sim 34000$ pseudoIFU cubes of 56 images.

\subsection{Challenge 4. Stellar Spectral Fitting}

The transportation of the calibration from J-PLUS (12 bands) to J-PAS (56 bands) will be done using high signal-to-noise stars whose SEDs will be fitted with spectra from libraries of stellar spectra (either theoretical or empirical). Then, the best fit stellar models will be convolved with the transmission curves of J-PAS to obtained the calibrated magnitudes of the stars in the J-PAS filter system. With several dozens of stars in each CCD of JPCamt, it will be possible to compute the photometric zero points for each exposure of J-PAS.

\subsection{Challenge 5. Spectro-übercalibration}

The survey strategy devised for J-PAS will ensure a high degree of overlapping between contiguous exposures. Following the ideas of Padmanabhan et al. (2008), we will used the information of the overlapping areas to improve the relative photometry in each band. However, we can push this idea further and using the synthetic photometry of many objects with flux calibrated spectra in the J-PAS area, we can tie the photometry between the different bands of J-PAS in a kind of spectro-übercalibration.

The photometric model that we will solved will contain $10^{7}$ zero points (one for each single exposure) and $\sim 10^{4} \delta Z P$, that will model the dependence of the $\mathrm{ZP}$ with the position in the CCD, the filter and the possible temporal variation of these coefficients, and the amount of data in the system will be of the order of $10^{9}$ measurements of stars.

\subsection{Challenge 6. Photometric Redshift}

With the calibrated photometry in all the J-PAS bands, the last step will be the computation of the phometric redshift. This will be done using the Bayesian approach implemented in BPZ (Benítez 2000), updated in BPZ2.0. It has been shown that the analysis of the residuals of the photo-z procedure in the different bands can help to detect offsets in the original photometry, that applied to all the objects can improve the final photometric redshifts (Molino et al. 2014).

\section{References}

Benítez, N. 2000, ApJ, 536, 571

Benítez, N., Dupke, R., Moles, M., et al. 2014, arXiv:1403.5237

Bertin, E. 2011, Astronomical Data Analysis Software and Systems XX, 442, 435

Hayes, D. S. \& Latham, D. W. 1975, ApJ, 197, 593

Molino, A., Benítez, N., Moles, M., et al. 2014, MNRAS, 441, 2891

Padmanabhan, N., Schlegel, D. J., Finkbeiner, D. P., et al. 2008, ApJ, 674, 1217

Taylor, K., Marín-Franch, A., Laporte, R., et al. 2014, Journal of Astronomical Instrumentation, 3,50010

Varela, J., Gruel, N., Cristóbal-Hornillos, D., et al. 2013, Highlights of Spanish Astrophysics VII, 957

$\dagger$ Javalambre Panoramic Camera. This is the camera that will be used to carried out JPAS (Taylor et al. 2014). 